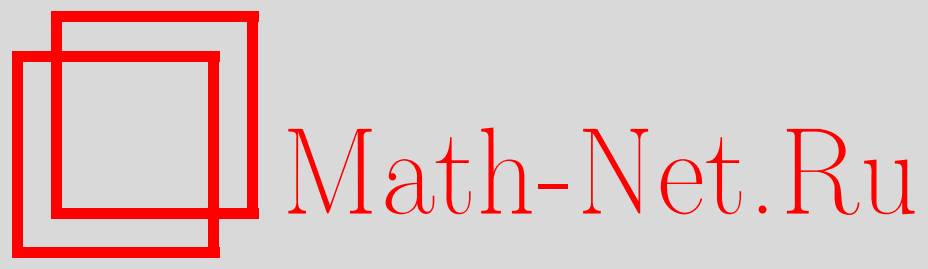

Л. Гаврилов, О числе предельных циклов, появляющихся при возмущении 2-седловых циклов векторных полей на плоскости, Функи. анализ и его прил., 2013, том 47, выпуск 3, 12-27

DOI: https://doi.org/10.4213/faa3118

Использование Общероссийского математического портала MathNet.Ru подразумевает, что вы прочитали и согласны с пользовательским соглашением http://www.mathnet.ru/rus/agreement

Параметры загрузки:

IP : 54.89 .56 .158

26 апреля 2023 г., 18:27:40

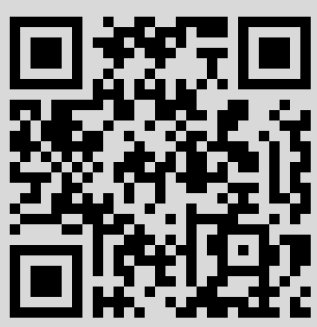




\title{
О числе предельных циклов, появляющихся при возмущении 2-седловых циклов векторных полей на плоскости
}

\author{
(c) 2013. Л. ГАВрилов
}

\begin{abstract}
Мы показываем, что число предельных циклов, рождающихся из 2-седловой петли аналитического векторного поля $X_{0}$ на плоскости посредством произвольного конечнопараметрического аналитического возмущения $X_{\lambda}, \lambda \in\left(\mathbb{R}^{N}, 0\right)$, равномерно ограничено по $\lambda$.
\end{abstract}

\section{§1. Введение}

Рассмотрим конечно-параметрическое аналитическое семейство аналитических векторных полей на плоскости

$$
X_{\lambda}=P(x, y, \lambda) \frac{\partial}{\partial x}+Q(x, y, \lambda) \frac{\partial}{\partial y}, \quad \lambda \in \mathbb{R}^{N},
$$

таких, что у $X_{0}$ есть предельное периодическое множество Г. Цикличность множества $\Gamma$ - это, грубо говоря, максимальное число предельных циклов векторного поля $X_{\lambda}$, стремящихся к $\Gamma$ при $\lambda \rightarrow 0$. Гипотеза Руссари о конечной цикличности гласит, что любое предельное периодическое множество, появляющееся в аналитическом конечно-параметрическом семействе аналитических векторных полей, имеет конечную иикличность [23]. Если эта гипотеза верна, то она влечет за собой конечность максимального числа $H(n)$ предельных циклов для полиномиального векторного поля степени $n$ на плоскости. Поэтому гипотеза Руссари играет фундаментальную роль во всех вопросах, связанных со второй частью 16-й проблемы Гильберта и ее разновидностями.

Напомним, что полициклом векторного поля $X_{0}$ называется топологический многоугольник, состоящий из сепаратрис и особых точек. Будем через $\Gamma_{k}$ обозначать $k$-седловой цикл векторного поля $X_{0}$ (или гиперболический $k$-график), т. е. полицикл, состоящий из $k$ различных седловых особых точек $p_{1}, \ldots, p_{k}, p_{k+1}$ $=p_{1}$ и сепаратрис (гетероклинических орбит), соединяющих $p_{i}$ с $p_{i+1}$, как на рис. 1. Такие $k$-седловые циклы, периодические орбиты и слабые фокусы или центры являются простейшими предельными периодическими множествами. Конечная цикличность периодических орбит и слабых фокусов хорошо известна и следует из теоремы Габриэлова [23, с. 68]. Конечная цикличность 1-седловой петли была доказана Руссари ([21], [22]).

Целью данной работы является доказательство конечной иикличности 2-седлового иикла при конечно-параметрическом аналитическом возмущении, см. теорему 4.

Некоторые частные случаи этого результата были доказаны ранее при различных условиях типичности на $X_{0}$ (или на семейство $X_{\lambda}$ ) Черкасом, Муртадой, Эль Морсалани, Дюмортье, Руссари, Руссо, Жебраном, Золадеком, Ли, 

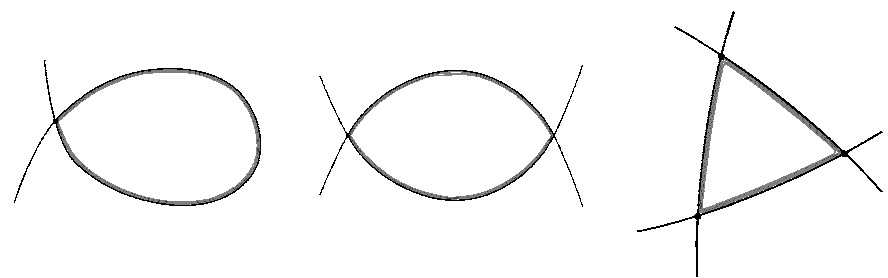

Рис. 1. Полициклы из одного, двух и трех седел.

Каубергом, Лука и другими ([6]-[8], [13], [16]); см. также [23, разд. 5.4.1], где имеется обзор результатов и ссылки до 1996 г. Конечная цикличность $k$-седлового цикла (при любом $k$ ) при конечно-параметрическом аналитическом возмущении была недавно анонсирована Муртадой [18].

Для типичных семейств векторных полей условие аналитичности можно ослабить. Как показали Ильяшенко и Яковенко [11] и Калошин [12], любой невырожденный элементарный полищикл, возникающий в типичном $k$-nараметрическом семействе $C^{\infty}$-гладких векторных полей, имеет конечную иикличность.

В отличие от отмеченных выше авторов, мы не будем использовать асимптотические ряды соответствующих отображений Дюлака. Вместо этого мы оцениваем количество предельных циклов вблизи $\Gamma_{2}$ в комплексной области, используя подходящий вариант принципа аргумента. Этот подход был предложен автором в [10], где изучалась цикличность гамильтоновых 2-седловых циклов. Хорошо известно, что предельные циклы систем на плоскости, близких к гамильтоновым, тесно связаны с нулями соответствующих абелевых интегралов, зависящих от параметра (см. так называемую ослабленную 16-ю проблему Гильберта в книге Арнольда [1, с. 360]). Нули полных эллиптических интегралов в комплексной области успешно изучались с помощью топологических методов (принцип аргумента) после новаторских работ Петрова [19], [20]; описание его метода см. также у Золадека [25, разд. 6]. Этот метод был использован в более общем контексте и в других работах, например, в [3], [2], а в [10] он использовался для того, чтобы заменить абелевы интегралы отображением первого возвращения Пуанкаре.

В данной работе мы найдем связь между неподвижными точками отображения первого возвращения Пуанкаре и неподвижными точками голономий сепаратрис седловых точек, которые соответствуют комплексным предельным циклам. Подсчет таких неподвижных точек сводится к вопросу о нулях семейств аналитических функиий, который несложно решается. Основным техническим инструментом является лемма 2, в которой мы показываем, что компоненты связности множества нулей мнимой части отображения Дюлака являются гладкими полуаналитическими кривыми. Этот факт позволяет оценить приращение аргумента отображения перемещения вдоль границы подходящей комплексной области, а затем применить принцип аргумента для подсчета нулей этого отображения в упомянутой области.

Заметим, что ранее связь монодромии и отображения Дюлака была использована Руссари для вычисления идеала Баутина, соответствующего отображению Дюлака [24]. Вместе с результатами из [21], [22] это также влечет за собой конечную цикличность 1-седловых циклов. 
Работа организована следующим образом. В 22 мы предоставляем необходимые технические сведения и доказываем основную техническую лемму 2. В $\S 3$ мы даем новое доказательство теоремы Руссари о конечной цикличности 1-седловых циклов. Происхождение нашего метода объяснено в 4 , где мы приводим краткое описание локальной версии так называемого трюка Петрова. Тот же метод легко приспосабливается в $\S 5$ для доказательства конечной цикличности множества $\Gamma_{2}$.

\section{§2. Отображение Дюлака}

Рассмотрим аналитическое семейство вещественных аналитических слоений на плоскости $\mathscr{F} \lambda, \lambda \in \mathbb{R}^{N}$, с невырожденной изолированной седловой особой точкой. Можно переместить эту точку в начало координат посредством подходящего параллельного переноса, аналитически зависящего от $\lambda$. Слоение $\mathscr{F} \lambda$ обладает двумя аналитическими сепаратрисами, трансверсально пересекающимися в седле и зависящими аналитически от $\lambda$ ([5], [17]). Поэтому после вещественной бианалитической замены переменных $x, y$, аналитически зависящей от $\lambda$, сепаратрисы отождествятся с осями координат $\{x=0\}$ и $\{y=0\}$, как на рис. 2; таким образом,

$$
\mathscr{F}_{\lambda}: x(1+\cdots) d y+\alpha(\lambda) y(1+\cdots) d x, \quad \alpha(0)>0,
$$

где отточие обозначает члены более высокого порядка по $x, y$ с коэффициентами, зависящими от $\lambda$. Число $\alpha(\lambda)$ - характеристическое число седла (отношение собственных значений). Далее мы будем полагать, что слоение (2) аналитическое и зависит аналитически от $\lambda$ в окрестности начала координат в $\mathbb{R}^{2} \times \mathbb{R}^{N}$.

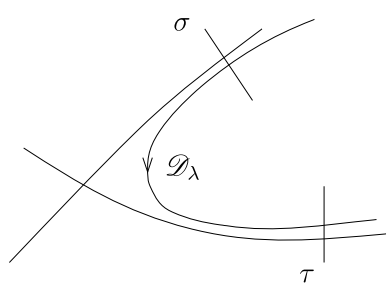

(i)

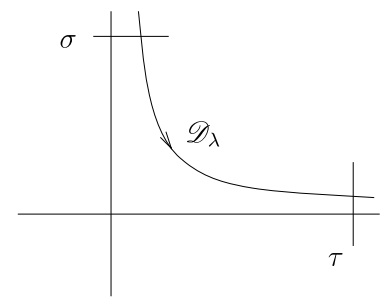

(ii)

Рис. 2. Отображение Дюлака.

Для достаточно малых $c_{1}, c_{2} \in \mathbb{R}$ рассмотрим открытые комплексные диски $\sigma \subset\left\{y=c_{1}\right\}, \tau \subset\left\{x=c_{2}\right\}$ с центрами в $\left(0, c_{1}\right)$ и $\left(c_{2}, 0\right)$ с координатами $x$ и $y$ соответственно. Вещественное отображение Дюлака есть росток в $x=0$ аналитического отображения

$$
\mathscr{D}_{\lambda}: \sigma \cap \mathbb{R}^{+} \rightarrow \tau \cap \mathbb{R}^{+}, \quad \mathscr{D}_{\lambda}(0)=0,
$$

определенного следующим образом: если $x \in \sigma \cap \mathbb{R}_{*}^{+}$, то $\mathscr{D}_{\lambda}(x) \in \tau \cap \mathbb{R}_{*}^{+}$есть пересечение листа $\gamma_{\lambda}(x)$ слоения (2), проходящего через $x$, с $\tau \cap \mathbb{R}_{*}^{+}$, см. рис. 2(ii). Такое геометрическое определение отображения $\mathscr{D}_{\lambda}$ позволяет в некоторой степени контролировать его аналитическое продолжение в комплексную область.

2.1. Аналитическое продолжение. Отображение Дюлака допускает аналитическое продолжение на некоторое зависящее от $\lambda$ открытое подмножество 
универсальной накрывающей $\sigma$ • проколотого диска $\sigma \backslash\{0\}$. Введем на $\sigma \bullet$ полярные координаты $\rho>0, \varphi \in \mathbb{R}, z=\rho \exp i \varphi$. Следующий результат широко известен (см., например, [10, приложение A]).

Теорема 1. Существует $\varepsilon_{0}>0$ и непрерьвная функиия

$$
\rho: \mathbb{R} \rightarrow \mathbb{R}_{*}^{+}, \quad \varphi \mapsto \rho(\varphi),
$$

такие, что отображсене Дюлака допускает аналитическое продолэсение в область

$$
\left\{(\lambda, \rho, \varphi) \in \mathbb{C}^{N} \times \sigma_{\bullet}:|\lambda|<\varepsilon_{0}, 0<\rho<\rho(\varphi)\right\} .
$$

Геометрическое содержание теоремы 1 состоит в следующем. Пусть $\left\{\gamma_{\lambda}(z)\right\}_{z, \lambda}$ есть непрерывное семейство путей, содержащихся в листах слоения $\mathscr{F} \lambda$ и соединяющих $z \in \sigma$ с $\tau$.

Если $z \in \sigma \cap \mathbb{R}_{*}^{+}$, то предположим, что $\gamma_{\lambda}(z)$ - вещественная орбита слоения $\mathscr{F}_{\lambda}$, содержащаяся в первом квадранте $x \geqslant 0, y \geqslant 0$ и соединяющая $z$ с $\tau$, см. рис. 2(ii). Приведенная выше теорема утверждает, что такое семейство орбит допускает продолжение до непрерывного семейства путей $\left\{\gamma_{\lambda}(z)\right\}_{z, \lambda}$, содержащихся в листах слоения $\mathscr{F} \lambda$ и соединяющих $z \in \sigma \bullet c \tau_{\bullet}$. Это семейство определено для всех $(\lambda, \rho, \varphi)$, принадлежащих области (3). Любой путь начинается в $z$ и заканчивается в единственной точке на $\sigma$, обозначаемой через $\mathscr{D} \lambda(z)$. Несмотря на то, что пути $\left\{\gamma_{\lambda}(z)\right\}_{z, \lambda}$ могут быть выбраны разными способами, их относительные гомотопические классы определены однозначно.

2.2. Монодромия отображения Дюлака и голономии сепаратрис. Сопоставим осям $\{x=0\},\{y=0\}$ с координатами $y$ и $x$ отображения голономии

$$
h_{\sigma}^{\lambda}: \sigma \rightarrow \sigma, \quad h_{\tau}^{\lambda}: \tau \rightarrow \tau,
$$

заданные двумя замкнутыми путями, содержащимися в осях $\{x=0\}$ и $\{y=0\}$ и начинающимися в $\left(0, c_{1}\right)$ и $\left(c_{2}, 0\right)$ соответственно. Договоримся, что любой замкнутый путь делает один оборот вокруг начала координат оси, в которой он содержится, в положительном направлении (напомним, что за координаты на этих осях мы выбрали $y$ и $x$ соответственно). Легко заметить, что в случае линейного слоения вида

$$
x d y+\alpha y d x=0, \quad \alpha \in \mathbb{R}^{+},
$$

мы имеем

$$
\mathscr{D}_{\alpha}: x \mapsto y=c_{1} c_{2}^{-\alpha} x^{\alpha}, \quad h_{\sigma}: x \mapsto x e^{-2 \pi i / \alpha}, \quad h_{\tau}: y \mapsto y e^{-2 \pi i \alpha} .
$$

В общем случае нелинейного слоения вида (2) отображение Дюлака $\mathscr{D} \lambda$ только асимптотически близко к $c_{1} c_{2}^{-\alpha} x^{\alpha}$, в то время как отображения голономии являются аналитическими по $x, y, \lambda$ и

$$
h_{\sigma}^{\lambda}: x \mapsto x e^{-2 \pi i / \alpha}+\ldots, \quad h_{\tau}^{\lambda}: y \mapsto y e^{-2 \pi i \alpha}+\ldots, \quad \alpha=\alpha(\lambda) .
$$

Отображение Дюлака $\mathscr{D}_{\lambda}$ есть трансцендентное многозначное отображение. Для $x>0$ будем обозначать через $\mathscr{D}_{\lambda}\left(e^{2 \pi i} x\right)$ результат аналитического продолжения отображения $\mathscr{D}_{\lambda}$ вдоль дуги радиуса $x$ и угла $2 \pi i$. Аналогично для $y>0$ обозначим через $\mathscr{D}_{\lambda}\left(e^{2 \pi i} y\right)$ результат аналитического продолжения отображения $\mathscr{D}_{\lambda}$ вдоль дуги радиуса $y$ и угла $2 \pi i$. 
Лемма 1. Для всех достаточно мальх $x>0, y>0 u|\lambda|$ имеют место равенства

$$
h_{\tau}^{\lambda} \circ \mathscr{D}_{\lambda}\left(e^{2 \pi i} x\right)=\mathscr{D}_{\lambda}(x), \quad h_{\sigma}^{\lambda} \circ \mathscr{D}_{\lambda}^{-1}\left(e^{2 \pi i} y\right)=\mathscr{D}_{\lambda}^{-1}(y) .
$$

Доказательство. Рассмотрим вместо $\mathscr{D}_{\lambda}$ соответствующий путь $\gamma_{\lambda}$. Петля $\gamma_{\lambda}\left(e^{2 \pi i} x\right)$ имеет то же начало, что и $\gamma_{\lambda}(x)$, поэтому можно взять их композицию, и получающаяся петля $\widetilde{\gamma}_{\lambda}(y)$ будет начинаться в $y=\mathscr{D}_{\lambda}\left(e^{2 \pi i} x\right) \in \tau$ и заканчиваться в $\mathscr{D}_{\lambda}(x) \in \tau$. В частном линейном случае $(4)$ с $\alpha=1$ слоение является расслоением, пути $\gamma_{\lambda}(\cdot)$ представляют собой относительные циклы в листах слоения $x y$, а путь $\widetilde{\gamma}_{\lambda}(y)$ замкнут и представляет собой исчезающий цикл. Утверждение леммы 1 есть тогда классическая формула ПикараЛефшеца. В общем случае результат получается «возмущением». Действительно, в линейном случае (4) с $\alpha=1$ семейство замкнутых путей $\left\{\widetilde{\gamma}_{\lambda}(y)\right\}_{y}$ определено для всех достаточно малых $y$ и $\widetilde{\gamma}_{\lambda}(0) \subset\{y=0\}$ есть замкнутый путь, обходящий один раз в положительном направлении начало координат на оси $\{y=0\}$. Заметим, что пути $\widetilde{\gamma}_{\lambda}(0)$ отделены от начала координат в $\mathbb{C}^{2}$. Отсюда следует, что $\widetilde{\gamma}_{\lambda}$ определяет голономию $h_{\tau}^{\lambda}$ сепаратрисы $\{y=0\}$, и это свойство сохраняется для любого достаточно малого возмущения слоения (4). Гомотетия $(x, y) \rightarrow(\varepsilon x, \varepsilon y)$ переводит слоение $(2)$ в малое возмущение линейного слоения (4), что завершает доказательство первого равенства (см. также [15]). Второе равенство леммы 1 доказывается сходным образом.

2.3. Множество нулей мнимой части отображения Дюлака. Рассмотрим универсальную накрывающую

$$
\mathbb{C} . \stackrel{\pi}{\rightarrow} \mathbb{C} \backslash\{0\}
$$

и множество нулей $\mathscr{H}_{\lambda} \subset \mathbb{C}$ • мнимой части отображения Дюлака $\mathscr{D}_{\lambda}$, соответствующего области (3),

$$
\mathscr{H}_{\lambda}=\left\{z=(\rho, \varphi) \in \mathbb{C}_{\bullet}: \operatorname{Im} \mathscr{D}_{\lambda}(z)=0,0<\rho<\rho(\varphi), \varphi \in \mathbb{R}\right\} .
$$

Таким образом, в случае линейного слоения (4) это множество нулей есть объединение полупрямых:

$$
\mathscr{H}_{\alpha}=\left\{z \in \mathbb{C}_{\bullet}: \operatorname{Im} z^{\alpha}=0\right\}=\bigcup_{k \in \mathbb{Z}} \mathscr{H}_{\alpha, k}, \quad \mathscr{H}_{\alpha, k}=\left\{(\rho, \varphi) \in \mathbb{C}_{\bullet}: \varphi=k \pi / \alpha\right\} .
$$

Чтобы описать $\mathscr{H}_{\lambda}$ для произвольного слоения вида (2) с характеристическим числом $\alpha(\lambda)>0$, рассмотрим ростки вещественных аналитических множеств в начале координат плоскости $\mathbb{R}^{2}=\mathbb{C}$

$$
C_{\lambda, k}=\left\{z \in \mathbb{C}=\mathbb{R}^{2}:\left(h_{\sigma}^{\lambda}\right)^{k}(z)=\bar{z}\right\},
$$

где $h_{\sigma}^{\lambda}$ есть отображение голономии, соответствующее сепаратрисе $\{x=0\}$.

Лемма 2. Множество нулей $\mathscr{H}_{\lambda} \subset \mathbb{C}$. мнимой части отображения Дюлака в области (3) есть объединение компонент связности $\mathscr{H}_{\lambda, k}$, заиндексированных с помощью $k \in \mathbb{Z}$.

- Каждое множество $C_{\lambda, k}$ вида (9) есть росток вещественной аналитической кривой в $\mathbb{R}^{2}$, гладкой в начале координат и касающейся в нем прямой

$$
\left\{z=s e^{i k \pi / \alpha(\lambda)}: s \in(\mathbb{R}, 0)\right\} .
$$


- Каждая компонента связности $\mathscr{H}_{\lambda, k}$ при проекции на плоскость $\mathbb{C}=\mathbb{R}^{2}$ отображением $\pi$ из (7) переходит в компоненту связности множества $C_{\lambda, k} \backslash$ $\{0\}$, касаюшуюся полупрямой (10), s> 0, в начале координат.

Замечание. Для произвольного биголоморфного отображения $h_{\sigma}^{\lambda}$, обращающегося в нуль в нуле, множество (9) совпадает с началом координат. Лемма 2 показывает, что, однако, для отображения монодромии $h_{\sigma}^{\lambda}$ седловой точки вещественно-аналитического векторного поля на плоскости множество $C_{\lambda, k}$ вида (9) есть росток вещественной аналитической кривой в $\mathbb{R}^{2}$, гладкий в начале координат. Расположение компонент связности множества $C_{\lambda, k} \backslash\{0\}$, касательных к полупрямым (10) с $s>0$, показано на рис. 3 .

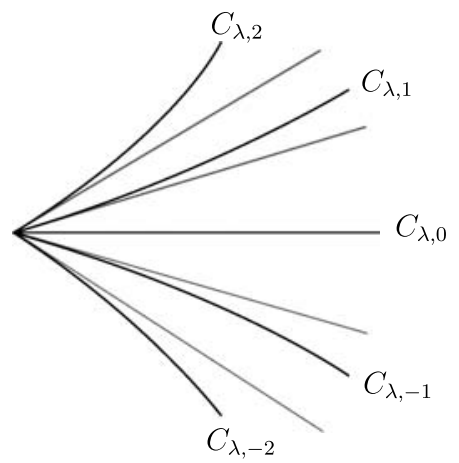

Рис. 3. Множество нулей $\mathscr{H}_{\lambda}$ мнимой части отображения Дюлака в проекции на комплексную плоскость $\mathbb{C}$.

Приведенная выше лемма есть главный технический результат данной работы. Аналитичность множества нулей $\mathscr{H}_{\lambda}$ отвечает за алгебраическое поведение отображения Дюлака.

Доказательство леммы 2. Пусть $x \in \sigma \cap \mathbb{R}^{+}$, и предположим, что $\mathscr{D}_{\lambda}\left(e^{i \varphi} x\right)$ $\in \mathbb{R}$ для некоторого $\varphi>0$. Поскольку отображение Дюлака вещественно вдоль $\sigma \cap \mathbb{R}^{+}$, то $\mathscr{D}_{\lambda}\left(e^{-i \varphi} x\right)$ является комплексно-сопряженным к $\mathscr{D}_{\lambda}\left(e^{i \varphi} x\right)$ и, значит,

$$
\mathscr{D}_{\lambda}\left(e^{-i \varphi} x\right)=\mathscr{D}_{\lambda}\left(e^{i \varphi} x\right) .
$$

Если точка $e^{-i \varphi} x$ рассматривается как прообраз точки $\mathscr{D}_{\lambda}\left(e^{-i \varphi} x\right)$ относительно действия отображения Дюлака $\mathscr{D}_{\lambda}^{-1}$, то точка $e^{i \varphi} x$ получается в результате аналитического продолжения отображения $\mathscr{D}_{\lambda}^{-1}$ вдоль подходящего замкнутого пути на $\tau$, начинающегося и заканчивающегося в $\mathscr{D}_{\lambda}\left(e^{-i \varphi} x\right)$. Если положить

$$
y=\mathscr{D}_{\lambda}\left(e^{-i \varphi} x\right), \quad e^{-i \varphi} x=\mathscr{D}_{\lambda}^{-1}(y),
$$

то $e^{ \pm i \varphi} x$ суть два значения многозначного отображения $\mathscr{D}_{\lambda}^{-1}(y)$ и, значит, по лемме 1 они отличаются на степень отображения монодромии $h_{\sigma}^{\lambda}$, т. е.

$$
\left(h_{\sigma}^{\lambda}\right)^{k}\left(e^{i \varphi} x\right)=e^{-i \varphi} x
$$

или, что эквивалентно,

$$
\left(h_{\sigma}^{\lambda}\right)^{k}(z)=\bar{z}, \quad z=e^{i \varphi} x, \quad \text { для некоторого } k \in \mathbb{Z} .
$$


Ясно, что каждое такое соотношение будет соответствовать компоненте связности $\mathscr{H}_{\lambda, k}$ множества $\mathscr{H}_{\lambda}$. Поскольку $\mathscr{H}_{\lambda, k}$ есть аналитическое множество вещественной размерности один, то $C_{\lambda, k}$ также есть аналитическое множество размерности один. Поэтому его можно определить одним из эквивалентных соотношений

$$
C_{\lambda, k} \subset\left\{z \in \mathbb{C}=\mathbb{R}^{2}: \operatorname{Re}\left[\left(h_{\sigma}^{\lambda}\right)^{k}(z)\right]=\operatorname{Re}(\bar{z})\right\}
$$

или

$$
C_{\lambda, k} \subset\left\{z \in \mathbb{C}=\mathbb{R}^{2}: \operatorname{Im}\left[\left(h_{\sigma}^{\lambda}\right)^{k}(z)\right]=\operatorname{Im}(\bar{z})\right\} .
$$

Поскольку $\frac{\partial}{\partial \bar{z}}\left[\left(h_{\sigma}^{\lambda}\right)^{k}(z)-\bar{z}\right]=-1$, линейная часть комплексно-аналитической функции

$$
\mathbb{R}^{2} \rightarrow \mathbb{C}, \quad(z, \bar{z}) \mapsto\left(h_{\sigma}^{\lambda}\right)^{k}(z)-\bar{z}
$$

не может быть тождественно равна нулю; поэтому $C_{\lambda, k} \subset \mathbb{R}^{2}$ есть вещественная аналитическая кривая, гладкая в начале координат. Из (6) следует, что проекция компоненты $\mathscr{H}_{\lambda, k}$ под действием $\pi$ на плоскость $\mathbb{C}=\mathbb{R}^{2}$ касается полупрямой (10), $s>0$, в начале координат.

2.4. Принцип аргумента. Пусть $\mathrm{D} \subset \mathbb{C}-$ предкомпактная область с кусочно-гладкой границей, и пусть $\psi: \mathbf{D} \rightarrow \mathbb{C}$ - аналитическая функция, непрерывно продолжающаяся на замыкание $\overline{\mathbf{D}}$ области $\mathbf{D}$. Обозначим через $Z_{\mathbf{D}}(\psi)$ количество нулей функции $\psi$ в $\mathbf{D}$, подсчитанных с кратностями. Если предположить, что $\psi$ не обращается в нуль на границе $\partial \mathbf{D}$, то приращение $\operatorname{Var}_{\partial \mathbf{D}}(\arg (\psi))$ аргумента функции $\psi$ вдоль ориентированной против часовой стрелки границы $\partial \mathbf{D}$ корректно определено и совпадает с индексом кривой $\psi(\partial \mathbf{D}) \subset \mathbb{C}$ вокруг начала координат. Классический принцип аргумента дает

$$
2 \pi Z_{\mathbf{D}}(\psi)=\operatorname{Var}_{\partial \mathbf{D}}(\arg (\psi)) .
$$

В более общей ситуации, если $\psi$ имеет нули на $\partial \mathbf{D}$, изолированные или нет, приращение аргумента $\operatorname{Var}_{\partial \mathbf{D}}(\arg (\psi))$ может не быть корректно определено.

Определение. Скажем, что $z \in \partial \mathbf{D}$ - регулярный нуль функции $\psi$, если $\psi(z)=0$ и $\psi$ допускает аналитическое продолжение в окрестность точки $z$ в $\mathbb{C}$.

Если мы предположим, что $\psi$ имеет только регулярные нули в $\overline{\mathbf{D}}$, то $\operatorname{Var}_{\partial \mathbf{D}}(\arg (\psi))$ корректно определено как сумма приращений аргумента огра-

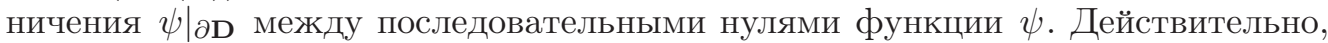
эти приращения конечны, поскольку граница $\partial D$ кусочно-гладкая. Принцип аргумента можно переформулировать следующим образом:

Предложение 1. Пусть $\mathbf{D} \subset \mathbb{C}$ - предкомпактное множество с кусочногладкой гранищей. Если $\psi: \overline{\mathbf{D}} \rightarrow \mathbb{C}$ - непрерьвная функиия, аналитическая в D и имеющая только регулярные нули в $\overline{\mathbf{D}}$, то

$$
2 \pi Z_{\mathbf{D}}(\psi) \leqslant \operatorname{Var}_{\partial \mathbf{D}}(\arg (\psi)) \leqslant 2 \pi Z_{\mathbf{D}}(\psi)+2 \pi Z_{\partial \mathbf{D}}(\psi) .
$$

Доказательство. Всегда существует многочлен $P$, такой, что $\psi / P$ не имеет нулей в $\overline{\mathbf{D}}$; поэтому достаточно проверять (12) только для многочленов. Множество D открыто, связно и ориентировано. Его граница кусочно-гладкая и потому не имеет самопересечений и обладает индуцированной ориентацией. Неравенство

$$
0 \leqslant \operatorname{Var}_{\partial \mathbf{D}}(\arg (z)) \leqslant 2 \pi
$$


позволяет «устранять» нули вдоль $\partial \mathbf{D}$, и, следовательно, из формулы (11) вытекает (12).

В данной работе первое неравенство в (12) будет использоваться для оценки числа нулей $Z_{\mathbf{D}}(\cdot)$. Для этого нам понадобятся оценки на приращение аргумента $\operatorname{Var}_{1}(\arg (\cdot))$ вдоль любого отрезка $l$ кривой. Более точно, пусть $l \subset \mathbb{R}^{2}=\mathbb{C}$ - отрезок гладкой вещественной аналитической кривой. Пусть $U \subset \mathbb{C}$ - открытое множество, содержащее $l$, и пусть $\psi_{\lambda}(z), \lambda \in\left(\mathbb{C}^{N}, 0\right)$, - росток в $\lambda=0$ семейства комплексно-аналитических функций в $U$. Для любого фиксированного $\lambda$, такого, что функция $\psi_{\lambda}$ не равна тождественно нулю, приращение $\mid \operatorname{Var}_{l}\left(\arg \left(\psi_{\lambda}\right) \mid\right.$ ее аргумента корректно определено.

Теорема 2. Пусть $l$ - отрезок вещественной аналитической кривой, $u$ пусть $\left\{\psi_{\lambda}\right\}_{\lambda}$ - семейство функиий, аналитических в окрестности этого отрезка и зависящих аналитически от $\lambda$. Тогда существует $\varepsilon_{0}>0$, такое, что

$$
\sup _{|\lambda|<\varepsilon_{0}, \psi_{\lambda} \neq 0} \mid \operatorname{Var}_{l}\left(\arg \left(\psi_{\lambda}\right) \mid<\infty\right.
$$

Этот результат вытекает из следующей теоремы Габриэлова ([14], [9]):

Теорема 3. Пусть $M, N$ - вещественные аналитические многообразия. Рассмотрим каноническую проекиию $\pi: M \times N \rightarrow N$. Для любого предкомпактного полуаналитического множества $E \subset M \times N$ число компонент связности прообразов $\pi^{-1}(n)$ равномерно ограничено сверху по $n \in N$.

Доказательство теоремы 2. Число изолированных нулей функции $\psi_{\lambda}$ вдоль $l$, подсчитанных с кратностями, равномерно ограничено по $\lambda$ при $\lambda=0$ (теорема Франсуаза-Йомдина [14]). На отрезке между двумя нулями функции $\psi_{\lambda}(\cdot)$ приращение аргумента, деленое на $2 \pi$, ограничено половиной числа нулей мнимой части функции $\psi_{\lambda}$ плюс сумма кратностей нулей функции $\psi_{\lambda}$ на концах этого отрезка. Мнимая часть функции $\psi_{\lambda}$ есть вещественно-аналитическая функция в $U \subset \mathbb{R}^{2}$, и из теоремы Габриэлова следует, что число компонент связности множества $\left\{\operatorname{Im}\left(\psi_{\lambda}\right)=0\right\} \cap l$ равномерно ограничено по $\lambda$ при $\lambda=0$.

\section{§3. Цикличность седловой петли}

Пусть $X_{\lambda}, \lambda \in\left(\mathbb{R}^{N}, 0\right)$, - росток аналитического семейства аналитических векторных полей на плоскости, таких, что $X_{0}$ имеет 1 -седловой цикл (гомоклиническую седловую петлю) $\Gamma_{1}$. Отображение первого возвращения, связанное с $\Gamma_{1}$, есть композиция отображения Дюлака $\mathscr{D} \lambda(z): \sigma \rightarrow \tau$ и отображения переноса $\mathscr{T}_{\lambda}(z)$, см. рис. 4. Мы предполагаем, что отображение Дюлака приведено к нормальной форме, как в разд. 2.1. Предельные циклы векторного поля $X_{\lambda}$ вблизи $\Gamma_{1}$ соответствуют нулям отображения перемещения

$$
\psi_{\lambda}(z)=\mathscr{D}_{\lambda}(z)-\mathscr{T}_{\lambda}(z)
$$

вблизи $z=0$. В подходящих локальных координатах на трансверсалях $\sigma$ и $\tau$ отображение переноса имеет вид $\mathscr{T}_{\lambda}(z) \equiv z$. Мы могли бы поступить иначе, просто выбрав $\sigma=\tau$ (не предполагая, что отображение Дюлака приведено к форме из разд. 2.1). Мы оценим число нулей функции $\psi_{\lambda}$ в области $\mathbf{D}_{R} \subset \mathbb{C}_{\bullet}$, ограниченной окружностью $\{\rho=R\}$ и компонентами связности $\mathscr{H}_{\lambda, 1}$ и $\mathscr{H}_{\lambda,-1}$ множества нулей мнимой части отображения Дюлака, как показано на рис. 5. Предположим, что $R>0$ настолько мало, что $\psi_{\lambda}(\cdot)$ аналитична в $\mathbf{D}_{R}$ для 
всех $\lambda \in \mathbb{R}^{N}$, таких, что $|\lambda| \leqslant \varepsilon_{0}$ (теорема 1 ), и аналитична даже на замыкании области $\mathbf{D}_{R}$, кроме, разумеется, точки $z=0$, где $\psi_{\lambda}(\cdot)$ только непрерывна. Действительно,

$$
\lim _{z \rightarrow 0, z \in \mathbf{D}_{R}} \mathscr{D}_{\lambda}(z)=0,
$$

в то время как $\mathscr{T}_{\lambda}(z)$ голоморфна в $z=0$, так что

$$
\lim _{z \rightarrow 0, z \in \mathbf{D}_{R}} \psi_{\lambda}(z)=c(\lambda),
$$

где $c(\lambda)$ аналитическая и $c(0)=0$. Если семейство функций $\psi_{\lambda}$ достаточно общее, то $c(\lambda) \not \equiv 0$, а в случае, когда $c(\lambda) \equiv 0$, мы можем заменить $\psi_{\lambda}$ на новое семейство $\psi_{\lambda}+\lambda_{N+1}, \lambda_{N+1} \in \mathbb{R}$, для которого предел в точке $z=0$ равен параметру $\lambda_{N+1}$. После такой подготовки мы можем доказать конечную цикличность гомоклинической петли $\Gamma_{1}$. Для этого мы применим предложение 1 (принцип аргумента) к семейству функций $\psi_{\lambda}$ в области $\mathbf{D}_{R}$. B процессе вычисления предполагается, что $R>0$ достаточно мало, $\varepsilon_{0}$ достаточно мало по сравнению с $R$ и $\lambda$ таково, что $|\lambda|<\varepsilon_{0}$. Для такого выбора параметров мы используем «физическое» обозначение

$$
0<|\lambda|<\varepsilon_{0} \ll R \ll 1 .
$$

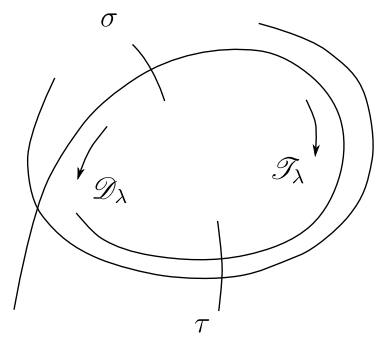

Рис. 4. Отображение Дюлака $\mathscr{D}_{\lambda}(z)$ и отображение переноса $\mathscr{T}_{\lambda}(z)$.
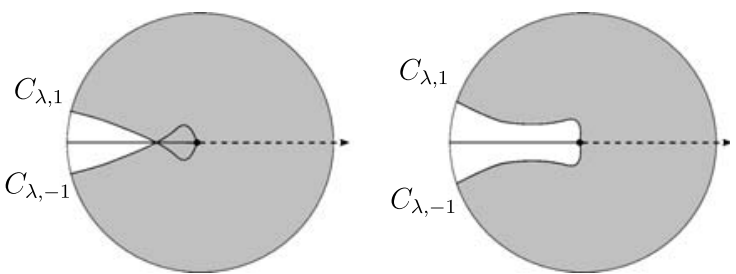

Рис. 5. Примеры областей $\mathbf{D}_{R} \subset \mathbb{C}$. в проекции на комплексную плоскость $\mathbb{C}$ под действием $\pi$ из (7).

Характеристическое число седловой точки не превосходит единицы только в подходящем полуаналитическом множестве пространства параметров и будет больше единицы в другом (дополнительном) полуаналитическом множестве. Поменяв, если нужно, местами $\sigma$ и $\tau$, мы будем также предполагать, что характеристическое число седловой точки не превосходит единицы для всех значений параметра. 
Приращение аргумента функции $\psi_{\lambda}$ равномерно ограничено по $\lambda$ вдоль окружности $\{z:|z|=R\}$ для угла, близкого или строго меньшего $2 \pi$ (теорема 2 ).

Вдоль кривой $C_{\lambda, 1}$ мнимая часть функции $\psi_{\lambda}$ совпадает с мнимой частью отображения переноса $-\mathscr{T}_{\lambda}(z)=-z$. Поэтому нули функции $\operatorname{Im}\left(\psi_{\lambda}\right)$ на $C_{\lambda, 1}$ это в точности точки пересечения кривой $C_{\lambda, 1}$ с интервалом $(-R, 0)$. По лемме 2

$$
C_{\lambda, 1} \cap \mathbb{R}=\left\{x \in \mathbb{R}: h_{\sigma}^{\lambda}(x)=x\right\}=C_{\lambda,-1} \cap \mathbb{R} .
$$

Поскольку $h_{\sigma}^{\lambda}(x)$ - аналитическое семейство аналитических функций, то по теореме Габриэлова число таких неподвижных точек равномерно ограничено по $\lambda$ на $[-R, 0]$. Нам осталось лишь проверить, что семейство $\left\{\psi_{\lambda}\right\}_{\lambda}$ имеет регулярные нули на границе области $\mathbf{D}_{R}$. Это так, когда $c(\lambda) \neq 0$, поскольку $\psi_{\lambda}(0)=c(\lambda)$. Таким образом, число изолированных нулей семейства функций

$$
\left\{\psi_{\lambda}: c(\lambda) \neq 0,|\lambda| \leqslant \varepsilon_{0}\right\}
$$

в области $\mathbf{D}_{R}$ ограничено некоторым целым числом $C$. Наконец, заметим, что условие $c(\lambda) \neq 0$ можно убрать. Действительно, если для некоторого $\lambda_{0},\left|\lambda_{0}\right| \leqslant$ $\varepsilon_{0}, c\left(\lambda_{0}\right)=0$, функция $\psi_{\lambda_{0}}$ имеет по крайней мере $C+1$ нулей в $\mathbf{D}_{R}$, то она имеет по крайней мере $C+1$ нулей в $\mathbf{D}_{R}$ в достаточно малой окрестности точки $\lambda_{0}$, что противоречит предыдущей оценке.

Итак, мы доказали следующий классический результат.

Теорема (Руссари [21], [22], [24]). Каждая гомоклиническая седловая петля (1-седловой чикл), появляющаяся в аналитическом конечно-параметрическом семействе аналитических векторных полей на плоскости, может породить не более чем конечное число предельных ииклов внутри семейства.

Заметим, что наш метод, точно так же как и теорема Руссари, позволяет более точно вычислить цикличность множества $\Gamma_{1}$. Мы не будем здесь вдаваться в подробности. Заметим лишь для иллюстрации этого факта, что если характеристическое число $\alpha(0)$ строго больше единицы, то суммарное приращение аргумента отображения перемещения вдоль границы области $\mathbf{D}_{R}$ строго меньше, чем $2 \pi$ (мы опускаем это вычисление), и цикличность множества $\Gamma_{1}$ равна нулю.

\section{§4. Трюк Петрова}

Этот параграф не является необходимым для доказательства главного результата, теоремы 4, но его цель - пролить некоторый свет на происхождение метода, использованного, чтобы оценить количество предельных циклов в окрестности седловой петли в предыдущем параграфе.

В обозначениях $\S 3$ рассмотрим аналитическое семейство аналитических векторных полей

$$
X_{\lambda}, \quad \lambda=\left(\lambda_{1}, \ldots, \lambda_{N}\right) \in\left(\mathbb{R}^{N}, 0\right),
$$

определяющих голоморфные слоения $\mathscr{F}_{\lambda}$ вида

$$
\mathscr{F}_{\lambda}=\left\{d H+\lambda_{1} \omega_{\lambda}=0\right\}, \quad \omega_{0} \neq 0,
$$

где $H$ - функция, а $\omega_{\lambda}$ - аналитическое семейство дифференциальных 1-форм. При этом и $H$, и $\omega_{\lambda}$ аналитичны в окрестности седловой петли $\Gamma_{1}$. Для определенности мы помещаем седловую точку в начало координат в $\mathbb{R}^{2}$, так что 
$d H(0)=0$. Далее будем считать, что седловая петля $\Gamma_{1}$ содержится в множестве уровня $\{H(x, y)=0\}$, а ее внутренность заполнена непрерывным семейством периодических орбит $\gamma_{0}(h) \subset\{H(x, y)=h\}$, параметризованных $h>0$, где $h=\left.H(x, y)\right|_{\sigma}$ есть ограничение функции $H$ на трансверсаль $\sigma$. Отображение перемещения аппроксимируется обычной формулой Пуанкаре-Понтрягина следующим образом:

$$
\psi_{\lambda}(h)=\lambda_{1} \int_{\gamma_{0}(h)} \omega_{\lambda}+o\left(\lambda_{1}\right),
$$

где $o\left(\lambda_{1}\right) / \lambda_{1}$ стремится к нулю при $\lambda$, стремящемся к нулю, равномерно по $h$ на каждом отрезке, на котором определено отображение перемещения. Нули функции $\psi_{\lambda}(\cdot)$ соответствуют предельным циклам, и, по крайней мере вдали от $\Gamma_{1} \subset\{H(x, y)=0\}$, они аппроксимируются нулями полного абелева интеграла

$$
h \mapsto I_{\lambda}(h)=\int_{\gamma_{0}(h)} \omega_{\lambda}, \quad h \geqslant 0 .
$$

Мы делаем предположение (на самом деле оправданное теоремой Руссари [21]), что это также верно в окрестности значения $h=0$ (соответствующего предельным циклам, близким к седловой петле $\left.\Gamma_{1}\right)$. Таким образом, имеет смысл доказать конечность максимального числа нулей абелева интеграла $I_{\lambda}(h)$, которые стремятся к $h=0$ при $\lambda$, стремящемся к началу координат пространства параметров. Это, конечно, следует из хорошо известного результата Варченко и Хованского. Однако мы будем использовать другую идею, предложенную Петровым [20], который показал, что аналогичная глобальная проблема для полных абелевых интегралов второго рода имеет алгебраическую природу. Петров использовал это наблюдение в нескольких статьях для определения точного числа нулей полных эллиптических интегралов и, следовательно, числа предельных циклов возмущенных гамильтоновых векторных полей; см., например, [25, разд. 6]. Мы готовы описать локальную версию метода Петрова.

Рассмотрим сектор

$$
S_{R}=\left\{z=\rho e^{i \varphi} \in \mathbb{C}: 0<\rho<R, 0<\varphi<2 \pi\right\} .
$$

Для фиксированного достаточно малого $R>0$ и всех достаточно малых $\|\lambda\|$ абелев интеграл $I_{\lambda}(z)$ допускает аналитическое продолжение на $S_{R}$. Чтобы оценить количество его нулей на $S_{R}$ (и, следовательно, на $\left.(0, R)\right)$, мы применяем принцип аргумента к области $S_{R}$. Вдоль окружности $\{\rho=R\}$ рост аргумента функции $I_{\lambda}$ равномерно ограничен по $\lambda$ (по теореме Габриэлова). Вдоль отрезка $[-R, 0]$ этот абелев интеграл допускает два аналитических продолжения $I_{\lambda}^{ \pm}(h)$. Поскольку $I_{\lambda}(\cdot)$ - вещественно-аналитическая функция на $(0, R)$, то

$$
I_{\lambda}^{+}(h)=\overline{I_{\lambda}^{-}(h)}, \quad h \in(-R, 0),
$$

и по формуле Пикара-Лефшеца

$$
2 \sqrt{-1} \operatorname{Im} I_{\lambda}^{+}(h)=I_{\lambda}^{+}(h)-I_{\lambda}^{-}(h)=\int_{\delta(h)} \omega_{\lambda}, \quad h \in(-R, 0),
$$

где $\delta(h) \subset\{H(x, y)=h\}$ - непрерывное семейство циклов, исчезающих в нуле при стремлении $h$ к нулю.

Таким образом, мнимая часть функции $I_{\lambda}(h)$ на $(-R, 0)$ является аналитической функцией и, вновь по теореме Габриэлова, ее нули равномерно ограничены 
по $\lambda$ на отрезке $[-R, 0]$. Отсюда следует равномерная ограниченность по $\lambda$ роста аргумента функции $I_{\lambda}(h)$ на $(-R, 0)$, что вместе с принципом аргумента доказывает конечность максимального числа нулей.

Доказательство конечной цикличности 1-седловой петли из предыдущего параграфа может восприниматься как обобщение метода Петрова. Действительно, формула Пикара-Лефшеца соответствует утверждению леммы 1, а по лемме 2 нули аналитического абелева интеграла (16) соответствуют неподвижным точкам (комплексным предельным циклам) отображения голономии $h_{\sigma}^{\lambda}$ сепаратрисы. Хорошо известно, что отображение голономии сепаратрисы аналитично, откуда и следует конечная цикличность седловой петли $\Gamma_{1}$.

\section{§5. Цикличность 2-седловых циклов}

Главным результатом работы является следующая

Теорема 4. Любая гетероклиническая седловая петля (2-седловой цикл), возникающий в аналитическом конечно-параметрическом семействе аналитических векторных полей на плоскости, порожсдает не более чем конечное число предельных ииклов внутри семейства.

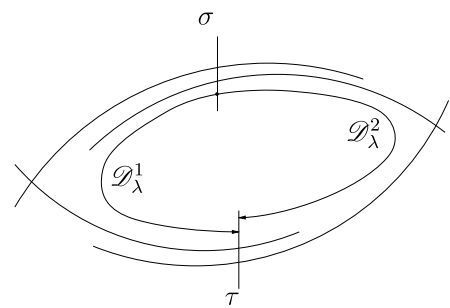

Рис. 6. Отображения Дюлака $\mathscr{D}_{\lambda}^{1}$ и $\mathscr{D}_{\lambda}^{2}$.

В обозначениях предыдущих параграфов предположим, что векторное поле $X_{0}$ имеет 2-седловую петлю $\Gamma_{2}$. Рассмотрим отображения Дюлака

$$
\mathscr{D}_{\lambda}^{i}: \sigma \rightarrow \tau, \quad i=1,2,
$$

связанные с соответствующим слоением, как на рис. 6. Каждое отображение $\mathscr{D}_{\lambda}^{i}$ есть композиция «локального» отображения Дюлака (как в §2) и двух вещественно-аналитических отображений переноса. Отсюда следует, что лемма 2 также применима к $\mathscr{D}_{\lambda}^{i}, i=1,2$. Всюду далее выбираем вещественноаналитическую локальную переменную $z$ на трансверсали $\sigma$, таким образом отождествляя $\sigma$ с открытым диском с центром в $0 \in \mathbb{C}$. Также будем предполагать, что $0=\sigma \cap \Gamma_{2}$. Функции $\mathscr{D}_{\lambda}^{i}(z), i=1,2$, многозначны на трансверсали $\sigma$ и имеют критические точки в $s_{i}(\lambda) \in \mathbb{R}, s_{i}(0)=0$. Функции $s_{i}$ вещественноаналитические. Предельные циклы векторного поля $X_{\lambda}$ вблизи $\Gamma_{2}$ соответствуют нулям отображения перемещения

$$
\psi_{\lambda}(z)=\mathscr{D}_{\lambda}^{1}(z)-\mathscr{D}_{\lambda}^{2}(z)
$$

вблизи $z=0$. Пусть $\alpha_{i}(\lambda)>0, i=1,2$, - характеристические числа седел. Мы будем предполагать, что (возможно, после перемены ролей $\sigma$ и $\tau$ ) $\alpha_{1}(0) \alpha_{2}(0) \geqslant 1$. Обозначим множества нулей мнимых частей отображений Дюлака $\mathscr{D}_{\lambda}^{1}(z)$ и $\mathscr{D}_{\lambda}^{2}(z)$ через $\mathscr{H}_{\lambda}^{1}$ и $\mathscr{H}_{\lambda}^{2}$ соответственно. Мы будем оценивать число 
нулей функции $\psi_{\lambda}$ в комплексной области $\mathbf{D}_{R}$ универсального накрытия множества $\mathbb{C} \backslash\left\{s_{1}(\lambda), s_{2}(\lambda)\right\}$, определенной следующим образом (без потери общности считаем, что $\left.s_{1}(\lambda) \leqslant s_{2}(\lambda)\right)$ :

- если $\alpha_{2}(0)>1$, область $\mathbf{D}_{R}$ ограничена окружностью

$$
S_{R}=\{z:|z|=R\},
$$

и кривыми

$$
\mathscr{H}_{\lambda, 1}^{1}, \mathscr{H}_{\lambda,-1}^{1}, \mathscr{H}_{\lambda, 1}^{2}, \mathscr{H}_{\lambda,-1}^{2},
$$

как показано на рис. 7;
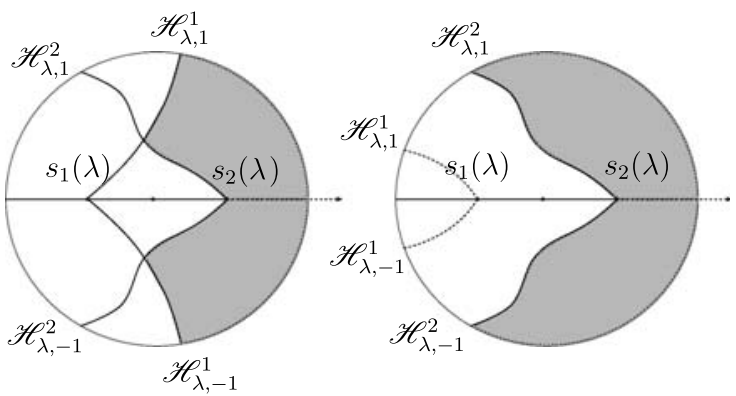

Рис. 7. Область $\mathbf{D}_{R} \subset \mathbb{C}$., спроектированная на комплексную плоскость $\mathbb{C}$, в случае $\alpha_{2}(0)>1$.

- если $\alpha_{2}(0) \leqslant 1$, то обязательно $\alpha_{1}(0) \geqslant 1$. Область $\mathbf{D}_{R}$ ограничена окружностью $S_{R}$, отрезком $\left[s_{1}(\lambda), s_{2}(\lambda)\right]$ и кривыми $\mathscr{H}_{\lambda, 1}^{1}, \mathscr{H}_{\lambda,-1}^{1}$, как показано на рис. 8.

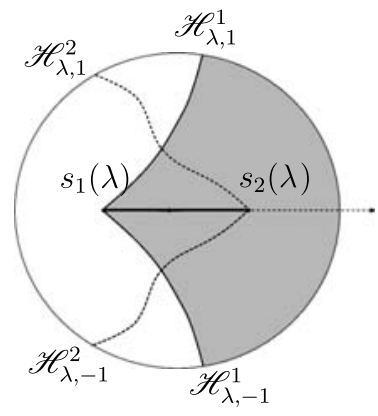

Рис. 8. Область $\mathbf{D}_{R} \subset \mathbb{C}$., спроектированная на комплексную плоскость $\mathbb{C}$, в случае $\alpha_{2}(0) \leqslant 1, \alpha_{1}(0) \geqslant 1$.

При доказательстве параметры $R$ и $\lambda$ будут выбраны, как в случае одного седла: константа $R$ будет достаточно малой, $\varepsilon_{0}>0$ будет достаточно мало по сравнению с $R$, а $\lambda \in \mathbb{R}^{N}$ будет таким, что $|\lambda|<\varepsilon_{0}$, см. (13). Как и в $\S 3$, мы будем предполагать без ограничения общности, что аналитические функции $c_{1}(\lambda), c_{2}(\lambda)$, где

$$
\lim _{z \rightarrow s_{1}(\lambda), z \in \mathbf{D}_{R}} \psi_{\lambda}(z)=c_{1}(\lambda), \quad \lim _{z \rightarrow s_{2}(\lambda), z \in \mathbf{D}_{R}} \psi_{\lambda}(z)=c_{2}(\lambda),
$$


не тождественно нулевые. Это гарантирует, что для типичных значений $\lambda$ отображение перемещения будет иметь лишь регулярные нули в замыкании области $\mathbf{D}_{R}$, так что можно применить принцип аргумента (предложение 1).

Доказательство теоремы 4. Из определения области $\mathbf{D}_{R} \subset \mathbb{C}$ • следует, что отображение перемещения $\psi_{\lambda}(z)$ аналитично в ней. Для подсчета нулей отображения перемещения (соответствующих вещественным и комплексным предельным циклам) в $\mathbf{D}_{R}$ мы применим предложение 1 (принцип аргумента) к семейству функций $\psi_{\lambda}$. Чтобы оценить приращение аргумента отображения перемещения вдоль границы области $\mathbf{D}_{R}$, мы повторяем рассуждения из 3.

Сначала рассмотрим случай $\alpha_{2}(0)>1$ (рис. 7). Компонента связности множества нулей мнимой части отображения $\mathscr{D}_{\lambda}^{2}$, касательная к прямой $\varphi=\pi / \alpha_{2}(\lambda)$, проходящей через $s_{2}(\lambda)$, пересекает окружность $S_{R}$ трансверсально, и вдоль этой окружности приращение аргумента функции $\psi_{\lambda}$ равномерно ограничено по $\lambda$ (теорема 2). Мнимая часть функции $\psi_{\lambda}(z)$, ограниченная на $\mathscr{H}_{\lambda}^{1}$, совпадает с мнимой частью отображения $-\mathscr{D}_{\lambda}^{2}$, и потому $\operatorname{Im} \psi_{\lambda}$ обращается в нуль на $\mathscr{H}_{\lambda, 1}^{1}, \mathscr{H}_{\lambda,-1}^{1}$ ровно в точках пересечения

$$
\mathscr{H}_{\lambda, 1}^{1} \cap \mathscr{H}_{\lambda, 1}^{2}, \quad \mathscr{H}_{\lambda,-1}^{1} \cap \mathscr{H}_{\lambda,-1}^{2} .
$$

Согласно лемме 2 , эти точки пересечения суть решения уравнения

$$
h_{2}^{\lambda}(z)=h_{1}^{\lambda}(z),
$$

где $h_{1}^{\lambda}, h_{2}^{\lambda}$ - голономии сепаратрис, пересекающих $\sigma$ и связанных с седловыми точками $s_{1}(\lambda)$ и $s_{2}(\lambda)$. По теореме Габриэлова число таких точек $z$ равномерно ограничено в диске $\{z:|z|<R\}$.

Теперь рассмотрим второй случай, когда $\alpha_{2}(0) \leqslant 1, \alpha_{1}(0) \geqslant 1$, см. рис. 8 . Вдоль окружности $S_{R}$ приращение аргумента функции $\psi_{\lambda}$ равномерно ограничено по $\lambda$ (теорема 2). Вдоль отрезка $\left[s_{1}(\lambda), s_{2}(\lambda)\right]$ мнимая часть отображения $\mathscr{D}_{\lambda}^{1}$ тождественно нулевая, а мнимая часть функции $\psi_{\lambda}(z)$, ограниченной на этот отрезок, совпадает с мнимой частью отображения $-\mathscr{D}_{\lambda}^{2}$. Поэтому нули функции $\operatorname{Im}\left(\psi_{\lambda}\right)$ вдоль $\left[s_{1}(\lambda), s_{2}(\lambda)\right]$ суть в точности точки пересечения кривой $\mathscr{H}_{\lambda, 1}^{2}$ с отрезком $\left[s_{1}(\lambda), s_{2}(\lambda)\right]$. По лемме 2 , как в $(14)$, эти точки пересечения суть решения уравнения

$$
h_{2}^{\lambda}(z)=z,
$$

где $h_{2}^{\lambda}$ - голономия сепаратрисы, пересекающей $\sigma$ и связанной с седловой точкой $s_{2}(\lambda)$. По теореме Габриэлова число таких неподвижных точек равномерно ограничено. Наконец, нули функции $\operatorname{Im}\left(\psi_{\lambda}\right)$ вдоль $\mathscr{H}_{\lambda, 1}^{1}$ и $\mathscr{H}_{\lambda,-1}^{1}$ оцениваются, как в случае $\alpha_{2}(0)>1$. Это завершает доказательство теоремы 4.

\section{§6. Заключительные замечания}

Равенство (18), определяющее комплексные предельные циклы, «ответственные» за цикличность двойной петли $\Gamma_{2}$, - главная новая составляющая в доказательстве по сравнению со случаем одного седла. В самом деле, решения уравнения (18) - это неподвижные точки отображения голономии $h_{2}^{\lambda} \circ\left(h_{1}^{\lambda}\right)^{-1}$, которое для $\lambda=0$ порождается замкнутой петлей $\gamma$, содержащейся в комплексифицированной сепаратрисе петли $\Gamma_{2}$, пересекающей трансверсаль $\sigma$. Топологический тип этой сепаратрисы вблизи $\Gamma_{2}$ есть диск с двумя проколами, 
соответствующими двум седлам $S_{1}(\lambda)$ и $S_{2}(\lambda)$. Ясно, что $\gamma$ делает один поворот вокруг каждого из них, но в зависимости от ориентации имеются две возможности, показанные на рис. 9(i), (ii). Простое вычисление на модельном примере показывает, что петля $\gamma$, связанная с голономией $h_{2}^{\lambda} \circ\left(h_{1}^{\lambda}\right)^{-1}$, имеет форму восьмерки, как показано на рис. 9(i). Читатель узнает в петле $\gamma$ ключевую составляющую доказательства локальной ограниченности числа нулей псевдоабелевых интегралов в [3], [4].

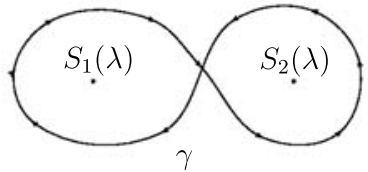

(i)

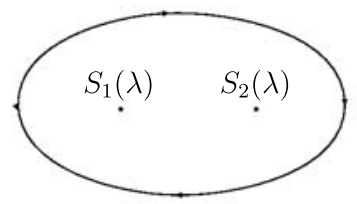

(ii)

Рис. 9. Петля $\gamma$ в форме восьмерки.

Несмотря на то, что теорема 4 - теорема существования, доказательство приводит к эффективным верхним оценкам на число рождающихся предельных циклов. Эта возможность исследуется в [10], где мы показываем, что цикличность гамильтоновой 2-седловой петли ограничена числом нулей пары соответствующих абелевых интегралов - явление, которое также объясняет появление посторонних предельных циклов в [8].

Стоит заметить, что наш результат о конечности верен (с тем же доказательством) и для других гиперболических полициклов (на плоскости или аналитической поверхности), например, показанных на рис. 10.
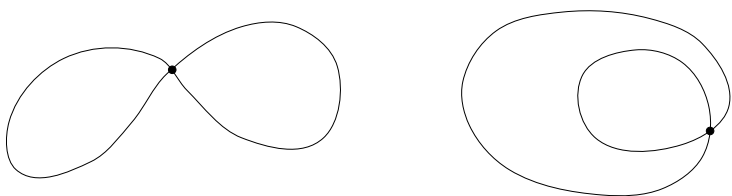

Рис. 10. Плоские гиперболические полициклы конечной цикличности.

Благодарности. Автор благодарит Марсина Бобьенского за полезные обсуждения, а также рецензентов за ценные замечания.

\section{ЛитерАТУРА}

[1] В. И. Арнольд, Геометрические методъ в теории обыкновенных дифберенциальных уравнений. 3-е изд., НИЦ «РХД», Ижевск, МЦНМО, Москва, 2002.

[2] G. Binyamini, D. Novikov, S. Yakovenko, On the number of zeros of Abelian integrals, Invent. Math., 181:2 (2010), 227-289.

[3] M. Bobieński, P. Mardešić, D. Novikov, Pseudo-Abelian integrals: unfolding generic exponential, J. Differential Equations, 247:12 (2009), 3357-3376.

[4] M. Bobieński, P. Mardešić, D. Novikov, Pseudo-Abelian integrals on slow-fast Darboux systems, http://arxiv.org/abs/1007.2001.

[5] Ch. Briot, J.-C. Bouquet, Recherches sur les propriétés des fonctions définies par des équations différentielles, J. Ecole Polytech., 21:36 (1856), 133-198. 
[6] M. Caubergh, F. Dumortier, R. Roussarie, Alien limit cycles in rigid unfoldings of a Hamiltonian 2-saddle cycle, Commun. Pure Appl. Anal., 6:1 (2007), 1-21.

[7] Л. А. Черкас, Об устойчивости особъх ииклов, Дифференциальные уравнения, 4 (1968), 1012-1017.

[8] F. Dumortier, R. Roussarie, Abelian integrals and limit cycles, J. Differential Equations, 227:1 (2006), 116-165.

[9] А. М. Габриэлов, О проекииях полуаналитических множеств, Функц. анализ и его прил., 2:4 (1968), 18-30.

[10] L. Gavrilov, On the number of limit cycles which appear by perturbation of hamiltonian two-saddle cycles of planar vector fields, Bull. Braz. Math. Soc., 42:1-23 (2011).

[11] Yu. Ilyashenko, S. Yakovenko, Finite cyclicity of elementary polycycles in generic families, in: Concerning the Hilber 16th Problem, Amer. Math. Soc. Transl., vol. 165, Amer. Math. Soc., Providence, RI, 1995, 21-95.

[12] V. Kaloshin, The existential Hilbert 16-th problem and an estimate for cyclicity of elementary polycycles, Invent. Math., 151:3 (2003), 451-512.

[13] C. Li, R. Roussarie, The cyclicity of the elliptic segment loops of the reversible quadratic Hamiltonian systems under quadratic perturbations, J. Differential Equations, 205:2 (2004), 488-520.

[14] S. Łojasiewicz, J.-Cl. Tougeron, M.-A. Zurro, Éclatement des coefficients des séries entières et deux théorèmes de Gabrielov, Manuscripta Math., 92:3 (1997), 325-337.

[15] F. Loray, Pseudo-groupe d'une singularité de feuilletage holomorphe en dimension deux, http://hal .archives-ouvertes.fr/hal-00016434/en/.

[16] S. Luca, F. Dumortier, M. Caubergh, R. Roussarie, Detecting alien limit cycles near a Hamiltonian 2-saddle cycle, Discrete Contin. Dyn. Syst., 25:4 (2009), 1081-1108.

[17] J.-F. Mattei, R. Moussu, Holonomie et intégrales premières, Ann. Sci. École Norm. Sup. (4), 13:4 (1980), 469-523.

[18] A. Mourtada, Action de derivations irreductibles sur les algebres quasi-regulieres d'Hilbert, http://arxiv.org/abs/0912.1560v1.

[19] Г. С. Петров, Эллиптические интеграль и их неколеблемость, Функц. анализ и его прил., 20:1 (1986), 46-49.

[20] Г. С. Петров, Вопрос о числе нулей эллиптического интеграла является полуалгебраическим, Матем. заметки, 44:3 (1988), 393-401.

[21] R. Roussarie, On the number of limit cycles which appear by perturbation of separatrix loop of planar vector fields, Bol. Soc. Brasil. Mat., 17:2 (1986), 67-101.

[22] R. Roussarie, Cyclicité finie des lacets et des points cuspidaux, Nonlinearity, 2:1 (1989), 73-117.

[23] R. Roussarie, Bifurcation of planar vector fields and Hilbert's sixteenth problem, Progress in Mathematics, vol. 164, Birkhäuser, Basel, 1998.

[24] R. Roussarie, Quasi-conformal mapping theorem and bifurcations, Bol. Soc. Brasil. Mat. (N.S.), 29:2 (1998), 229-251.

[25] H. Żoładek, The Monodromy Group, Mathematics Institute of the Polish Academy of Sciences. Math. Monographs (New Series), vol. 67, Birkhäuser, Basel, 2006.

Institut de Mathématiques de Toulouse email: lubomir.gavrilov@math.univ-toulouse.fr 